\title{
Application of Atom Probe Tomography to Complex Microstructures of Laser Additively Manufactured Samples
}

\author{
Philipp Kürnsteiner ${ }^{*}$, Avinash Hariharan ${ }^{1}$, Hyo Yun Jung ${ }^{1}$, Nicolas Peter ${ }^{1}$, Markus Benjamin Wilms ${ }^{2}$, \\ Andreas Weisheit ${ }^{2}$, Pere Barriobero-Vila ${ }^{3}$, Baptiste Gault ${ }^{1}$, Dierk Raabe ${ }^{1}$ and Eric Aimé Jägle ${ }^{1}$ \\ 1. Max-Planck-Institut für Eisenforschung GmbH, Düsseldorf 40237, Germany \\ 2. Fraunhofer Institute for Laser Technology ILT, Aachen 52074, Germany \\ 3. German Aerospace Center (DLR), 51147 Cologne, Germany \\ * Corresponding author: p.kuernsteiner@mpie.de
}

Additive Manufacturing (AM) technologies have gained increasing interest across multiple industrial sectors ranging from biomedical to aerospace. AM is not only used for prototyping, but also for tooling as well as for final part production. The computer-controlled, layer-by-layer building up process allows for increased design freedom enabling to produce almost any shape. Additional benefits are potential resource efficiency, increased product customization and lightweight design. Two prominent metalbased laser AM (LAM) techniques are laser powder bed fusion (LPBF) and laser metal deposition (LMD). In LPBF, a focused laser beam is scanned over a bed filled with metal powder to locally melt and fuse the powder to produce fully dense metal parts. The next layer of powder is then distributed and the laser scans again. LMD is a nozzle-based AM process in which a focused laser beam creates a melt pool in the build's surface. Metallic powder is then injected into the melt pool through a nozzle. The 3D part is built by moving the nozzle/laser assembly forward track by track and layer by layer.

Material produced by LAM exhibits a unique thermal history: initially, the material is cooled rapidly from the liquid state in the meltpool. Subsequently, the material experiences a cyclic reheating, the socalled intrinsic heat treatment (IHT), as neighboring tracks and further layers are deposited during the LAM process. Consequences of this thermal history are very complex, sometimes hierarchical microstructures with inhomogeneities at scales ranging from nanometers up millimeters. Fully understanding and characterizing these microstructures is challenging and requires the combination of methods spanning a similar range: from light optical microscopy (LOM) to electron microscopy to atom probe tomography (APT). Here we present examples how APT can deliver valuable information on complex microstructures to better understand the IHT, rapid solidification as well as phase separation in different metallic alloys. Here I will discuss examples in steel, Al- and Ni-based superalloys, and high entropy alloys (HEA).

The IHT can be exploited to trigger precipitation reactions already during the manufacturing process [1]. This way, it is possible to produce in-situ hardened parts that do not need to undergo a post-process aging heat treatment. This effect also opens new opportunities for designing alloys that are tailor-made for LAM processes. APT coupled with synchrotron high-energy X-Ray diffraction (HEXRD) has proven to be an indispensable tool to characterize the nanometer-sized NiAl precipitates that are formed in maraging steels. The chemistry of the extremely high number densities of up to $10^{25} \mathrm{NiAl}$ precipitates per $\mathrm{m}^{3}$ would be challenging to analyze with other high-resolution techniques. In Al-Sc alloys, $\mathrm{Al}_{3}(\mathrm{Sc}, \mathrm{Zr})$ precipitation can be triggered by the IHT. APT is well suited for analyzing the $\mathrm{Zr}$ shell of the coherent precipitates, precipitates sizes and number densities as well as matrix compositions. Additionally, we can study details of the interface of large, $\mu \mathrm{m}$ sized $\mathrm{Al}_{3}(\mathrm{Sc}, \mathrm{Zr})$ particles with the $\mathrm{Al}$ matrix [2]. 
Recently, there have been increasing reports that the LPBF produced HEAs have homogeneous elemental distributions owing to the rapid melting and solidification rate $\left(10^{4} \sim 10^{6} \mathrm{~K} / \mathrm{s}\right)$ [3]. Except for $\mathrm{Mn}$, equi-atomic $\mathrm{AlCoCrFeMnNi}$ seemed to have homogeneous elemental distribution when investigated at length scales that can be probed by scanning electron microscopy (SEM). However, high resolution analysis using APT and scanning transmission electron microscopy (STEM) revealed that the LPBF specimen consisted of a nano-scaled modulated structure of Ni/A1 rich $\mathrm{B} 2$ and $\mathrm{Cr} / \mathrm{Fe}$ rich $\mathrm{A} 2$ phase (Figure 1). Such a microstructure offers potentially promising mechanical properties. These results introduce a new concept for direct synthesis of nanostructured spinodal HEAs.

A key issue, preventing widespread application of LPBF to certain polycrystalline nickel-base superalloys, is the formation of micro-cracks during the process. These are solidification cracks that occur during the last stage of solidification [4] as a result of micro-segregation of solute elements to the interdendritic liquid. APT studies coupled with targeted sample preparation and transmission Kikuchi diffraction (TKD) was used to quantify the chemical composition profile of the solutes across grain boundaries with unambiguously identified misorientation. It was possible to reveal a different solute enrichment profile in the low-angle and high-angle grain boundaries. These results can be used to rationalize the contribution of micro-segregation to solidification cracking.

APT has proved itself to be an extremely powerful technique to study metallic AM samples, especially when combined with LOM and SEM to cover larger length scales as well as HEXRD and STEM delivering structural information to be combined with the chemical information from APT [5].

\section{References:}

[1] P. Kürnsteiner et al., Acta Mater. 129 (2017), 52-60.

[2] B. Gault et al., J. Mater. Res. 33 (2018), 4018-4030.

[3] R. Zhou et al., Intermetallics 94 (2018), 165-171.

[4] M. Rappaz, A. Jacot and W.J. Boettinger, Mater. Trans. A. 34 (2003), 467-479.

[5] This work was funded by the MPG and FhG through the AProLAM project, the DFG through the PaCCman-Project (SPP 2006) and IMPRS-Surmat. The authors are grateful to Uwe Tezins and Andreas Sturm for their support to the FIB and APT facilities at MPIE.
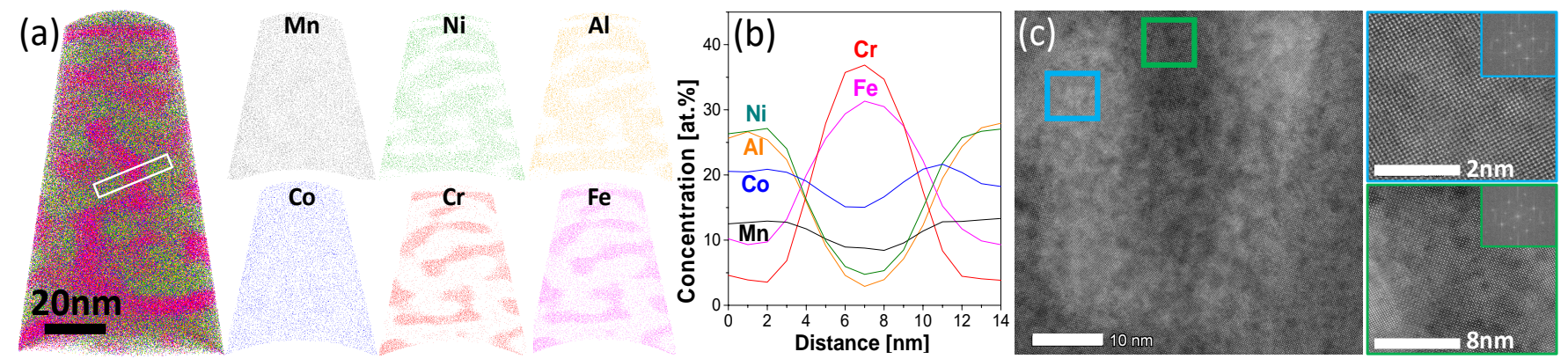

Figure 1. Figure 1 (a) and (b) show the three-dimensional elemental distribution and 1D-composition profile from the white box. STEM-HAADF analysis shown in figure 1 (c) reveals that the Ni-Al rich and $\mathrm{Cr}-\mathrm{Fe}$ rich region having coherent interphase boundary are ordered $\mathrm{BCC}$ and disordered $\mathrm{BCC}$ phase, respectively. 